\title{
A Quartic Subdomain Finite Element Method for the Modified KdV Equation
}

\author{
Seydi Battal Gazi Karakoc* \\ Department of Mathematics, Nevsehir Haci Bektas Veli University, Turkey
}

\begin{abstract}
In this article, we have obtained numerical solutions of the modified Korteweg-de Vries (MKdV) equation by a numerical technique attributed on subdomain finite element method using quartic B-splines. The proposed numerical algorithm is controlled by applying three test problems including single solitary wave, interaction of two and three solitary waves. To inspect the performance of the newly applied method, the error norms, $L_{2}$ and $L_{\infty}$, as well as the four lowest invariants, $I_{1}, I_{2}, I_{3}$ and $I_{4}$ have been computed. Linear stability analysis of the algorithm is also examined.
\end{abstract}

Keywords Modified Korteweg-de Vries equation, finite element method, subdomain, quartic B-spline, soliton.

AMS 2010 subject classifications 65N30, 65D07, 74S05,74J35, 76B25

DOI: $10.19139 /$ soic.v6i4.485

\section{Introduction}

The modified Korteweg de-Vries (MKdV) equation which will be studied in this article is related to the following Korteweg de-Vries (KdV) equation

$$
U_{t}+\varepsilon U U_{x}+\mu U_{x x x}=0 .
$$

The terms $U U_{x}$ and $U_{x x x}$ in the Eq.(1) represent the nonlinear convection and dispersion, respectively. $\mathrm{KdV}$ equation is one of the main mathematical model for describing the theory of water waves in shallow channels. Some important physical phenomena for example propagation of long waves in shallow water waves, bubbleliquid mixtures, ion acoustic plasma waves and wave phenomena in enharmonic crystals can be described by the KdV equation which was first suggested by Korteweg and de Vries [1]. The exact solutions of the equation obtained by [2,3]. At first, Zabusky and Kruskal solved the KdV equation numerically using the finite difference method [4]. Gardner et al. [5] showed the existence and uniqueness of solutions of the KdV equation. Many researches have used various numerical methods including finite difference method $[6,7]$, finite element method [8-18], pseudospectral method [3] and heat balance integral method [19] to solve the equation. MKdV equation is a special case of the generalized Korteweg de-Vries (GKdV) equation having the form

$$
U_{t}+\varepsilon\left(U^{p}\right)_{x}+\mu U_{x x x}=0,
$$

where $p$ is a positive integer. In this study, we will consider the MKdV equation, a special form of (2) with the choice $p=2, \varepsilon=3$ and $\mu=1$,

\footnotetext{
*Correspondence to: Seydi Battal Gazi Karakoc (Email: sbgkarakoc@ nevsehir.edu.tr). Department of Mathematics, Faculty of Science and Art, Nevsehir Haci Bektas Veli University, Nevsehir, 50300, Turkey.
}

ISSN 2310-5070 (online) ISSN 2311-004X (print)

Copyright (C) 2018 International Academic Press 


$$
U_{t}+3 U^{2} U_{x}+U_{x x x}=0
$$

with the homogeneous boundary conditions

$$
\begin{array}{ll}
U(a, t)=0, & U(b, t)=0, \\
U_{x}(a, t)=0, & U_{x}(b, t)=0, \quad t>0
\end{array}
$$

and an initial condition

$$
U(x, 0)=f(x) \quad a \leq x \leq b
$$

where $t$ is time, $x$ is the space coordinate and $f(x)$ is a detected function. MKdV equation have a limited number of numerical studies in the literature. Kaya [20], used the Adomian decomposition method to obtain the higher order modified Korteweg de-Vries equation with initial condition. MKdV equation have been solved by using Galerkins' method with quadratic B-spline finite elements by Biswas et al. [21]. Raslan and Baghdady [22, 23], showed the accuracy and stability of the difference solution of the MKdV equation and they obtained the numerical aspects of the dynamics of shallow water waves along lakes' shores and beaches modeled by the MKdV equation. A new variety of $(3+1)$-dimensional MKdV equation and multiple soliton solutions for each new equation were established by Wazwaz [24, 25]. A lumped Galerkin and Petrov Galerkin methods were applied to the MKdV equation by Ak et al. [26, 27].

In the present study, we have developed Subdomain finite element method for the MKdV equation using quartic B-spline functions. Motion of a single solitary wave, interaction of two and three solitary waves are examined to show the performance and efficiency of the suggested method. We show that the suggested method is unconditionally stable applying the von-Neumann stability analysis.

\section{Quartic B-splines}

Quartic B-splines $\phi_{m}(x),(m=-2,-1, \ldots, N, N+1)$ at the knots $x_{m}$ are designated over the interval $[a, b]$ by Prenter [28]

$$
\phi_{m}(x)=\frac{1}{h^{4}} \begin{cases}\left(x-x_{m-2}\right)^{4}, & {\left[x_{m-2}, x_{m-1}\right]} \\ \left(x-x_{m-2}\right)^{4}-5\left(x-x_{m-1}\right)^{4}, & {\left[x_{m-1}, x_{m}\right]} \\ \left(x-x_{m-2}\right)^{4}-5\left(x-x_{m-1}\right)^{4}+10\left(x-x_{m}\right)^{4}, & {\left[x_{m}, x_{m+1}\right]} \\ \left(x_{m+3}-x\right)^{4}-5\left(x_{m+2}-x\right)^{4}, & {\left[x_{m+1}, x_{m+2}\right]} \\ \left(x_{m+3}-x\right)^{4}, & {\left[x_{m+2}, x_{m+3}\right]} \\ 0 & \text { elsewhere. }\end{cases}
$$

Each quartic B-spline covers five elements, thus each element $\left[x_{m}, x_{m+1}\right]$ is covered by five B-splines. A typical finite interval $\left[x_{m}, x_{m+1}\right]$ is mapped to the interval $[0,1]$ by a local coordinate transformation defined by $h \xi=x-x_{m}, 0 \leq \xi \leq 1$. So quartic B-splines (6) in terms of $\xi$ over $[0,1]$ can be given as follows:

$$
\begin{aligned}
& \phi_{m-2}=1-4 \xi+6 \xi^{2}-4 \xi^{3}+\xi^{4}, \\
& \phi_{m-1}=11-12 \xi-6 \xi^{2}+12 \xi^{3}-\xi^{4}, \\
& \phi_{m}=11+12 \xi-6 \xi^{2}-12 \xi^{3}+\xi^{4}, \\
& \phi_{m+1}=1+4 \xi+6 \xi^{2}+4 \xi^{3}-\xi^{4}, \\
& \phi_{m+2}=\xi^{4} .
\end{aligned}
$$


For the problem, the finite elements are identified with the interval $\left[x_{m}, x_{m+1}\right]$. Using Eq.(6) and Eq.(7), the nodal values of $U_{m}, U_{m}^{\prime}, U_{m}^{\prime \prime}$ and $U_{m}^{\prime \prime \prime}$ are given in terms of the element parameters $\delta_{m}$ by

$$
\begin{aligned}
& U_{N}\left(x_{m}, t\right)=U_{m}=\delta_{m-2}+11 \delta_{m-1}+11 \delta_{m}+\delta_{m+1}, \\
& U_{m}^{\prime}=\frac{4}{h}\left(-\delta_{m-2}-3 \delta_{m-1}+3 \delta_{m}+\delta_{m+1}\right), \\
& U_{m}^{\prime \prime}=\frac{12}{h^{2}}\left(\delta_{m-2}-\delta_{m-1}-\delta_{m}+\delta_{m+1}\right), \\
& U_{m}^{\prime \prime \prime}=\frac{44}{h^{3}}\left(-\delta_{m-2}+2 \delta_{m-1}-2 \delta_{m+1}+\delta_{m+2}\right)
\end{aligned}
$$

and the variation of $U$ over the element $\left[x_{m}, x_{m+1}\right]$ is given by

$$
U=\sum_{j=m-2}^{m+2} \phi_{j}(\xi) \delta_{j}(t) .
$$

\section{Subdomain Method For The MKdV Equation}

For the numerical algorithm, solution area of the problem is limited over an interval $a \leq x \leq b$. Let the partition of the space interval $[a, b]$ into equally sized finite elements of length $h$ at the points $x_{m}$ like that $a=x_{0}<x_{1}<\ldots<$ $x_{N}=b$ and $h=\frac{b-a}{N}$. The set of quartic B-spline functions $\left\{\phi_{-2}(x), \phi_{-1}(x), \ldots, \phi_{N}(x), \phi_{N+1}(x)\right\}$ form a basis over the solution region $[a, b]$. The numerical solution $U_{N}(x, t)$ is expressed in terms of the quartic $\mathrm{B}$-splines as

$$
U_{N}(x, t)=\sum_{m=-2}^{N+1} \phi_{m}(x) \delta_{m}(t)
$$

where $\delta_{m}(t)$ are time dependent parameters and will be defined from the both boundary and weighted residual conditions. Applying the numerical approach to Eq.(3) with the weight function

$$
W_{m}(x)=\left\{\begin{array}{cc}
1, & x \in\left[x_{m}, x_{m+1}\right] \\
0, & \text { otherwise }
\end{array}\right\}
$$

we get the following equation

$$
\int_{x_{m}}^{x_{m+1}} 1 .\left(U_{t}+3 U^{2} U_{x}+U_{x x x}\right) d x=0 .
$$

When we use the $h \xi=x-x_{m}$ transformation into the weak form of Eq.(12) and integrating it term by term with some regulation by parts, guides to

$$
\begin{aligned}
& \frac{h}{5}\left(\dot{\delta}_{m-2}+26 \dot{\delta}_{m-1}+66 \dot{\delta}_{m}+26 \dot{\delta}_{m+1}+\dot{\delta}_{m+2}\right)+Z_{m}\left(-\delta_{m-2}-10 \delta_{m-1}+10 \delta_{m+1}+\delta_{m+2}\right)+ \\
& \mu \frac{12}{h^{2}}\left(-\delta_{m-2}+2 \delta_{m-1}-2 \delta_{m+1}+\delta_{m+2}\right)=0,
\end{aligned}
$$

where the dot denotes differentiation with respect to $t$ and

$$
Z_{m}=U_{m}=3\left(\delta_{m-2}+11 \delta_{m-1}+11 \delta_{m}+\delta_{m+1}\right)^{2} .
$$

If time parameters $\delta_{i}$ and its time derivatives $\dot{\delta}_{i}$ in Eq.(13) are discretized by the Crank-Nicolson formula and usual forward difference approach respectively,

$$
\delta_{i}=\frac{\delta_{i}^{n+1}+\delta_{i}^{n}}{2}, \quad \dot{\delta}_{i}=\frac{\delta_{i}^{n+1}-\delta_{i}^{n}}{\Delta t}
$$

we derive a repetition relationship between two time levels $n$ and $n+1$ relating two unknown parameters $\delta_{i}^{n+1}, \delta_{i}^{n}$ for $i=m-2, \ldots, m+2$ 


$$
\gamma_{1} \delta_{m-2}^{n+1}+\gamma_{2} \delta_{m-1}^{n+1}+\gamma_{3} \delta_{m}^{n+1}+\gamma_{4} \delta_{m+1}^{n+1}+\gamma_{5} \delta_{m+2}^{n+1}=\gamma_{5} \delta_{m-2}^{n}+\gamma_{4} \delta_{m-1}^{n}+\gamma_{3} \delta_{m}^{n}+\gamma_{2} \delta_{m+1}^{n}+\gamma_{1} \delta_{m+2}^{n}
$$

where

$$
\begin{aligned}
& \gamma_{1}=\left[1-E Z_{m}-M\right], \quad \gamma_{2}=\left[26-10 E Z_{m}+2 M\right], \gamma_{3}=[66], \\
& \gamma_{4}=\left[26+10 E Z_{m}-2 M\right], \gamma_{5}=\left[1+E Z_{m}+M\right], \quad m=0,1, \ldots, N-1, \\
& E=\frac{5}{2 h} \varepsilon \Delta t, \quad M=\frac{30}{h^{3}} \mu \Delta t .
\end{aligned}
$$

The system (16) involves of $N$ linear equations containing $(N+4)$ unknown coefficients $\left(\delta_{-2}, \delta_{-1}, \ldots, \delta_{N}, \delta_{N+1}\right)^{T}$. We need four additional restraints to obtain a unique solution for this system. These are obtained from the boundary conditions (4) and can be used to remove $\delta_{-2}, \delta_{-1}, \delta_{N}$ and $\delta_{N+1}$ from the systems (16) which occures a matrix equation for the $N$ unknowns $d^{n}=\left(\delta_{0}, \delta_{1}, \ldots, \delta_{N-1}\right)^{T}$ of the form

$$
T \mathbf{d}^{\mathbf{n}+\mathbf{1}}=V \mathbf{d}^{\mathbf{n}} .
$$

This matrix equation have been solved by using a variant of the Thomas algorithm. However, two or three inner iterations are implemented to the term $\delta^{n *}=\delta^{n}+\frac{1}{2}\left(\delta^{n}-\delta^{n-1}\right)$ at each time step to overcome the non-linearity caused by $Z_{m}$. Before the solution process begins iteratively, the initial vector $d^{0}$ must be established by using the initial condition and following derivatives at the boundaries;

$$
\begin{aligned}
\left(U_{N}\right)_{x}(a, 0) & =0, & & \left(U_{N}\right)_{x}(b, 0)=0, \\
\left(U_{N}\right)_{x x}(a, 0) & =0, & & \left(U_{N}\right)_{x x}(b, 0)=0 .
\end{aligned}
$$

So we have the following matrix form for the initial vector $d^{0}$;

$$
V d^{0}=w,
$$

$$
\begin{aligned}
& \text { where } V=\left[\begin{array}{ccccccccc}
18 & 6 & & & & & \\
11.5 & 11.5 & 1 & & & & & \\
1 & 11 & 11 & 1 & & & & \\
& & & & 1 & 11 & 11 & 1 \\
& & & & 2 & 14 & 8
\end{array}\right] \\
& d^{0}=\left(\delta_{0}, \delta_{1}, \delta_{2}, \ldots, \delta_{N-2}, \delta_{N-1}, \delta_{N}\right)^{T} \text { and } w=\left(U\left(x_{0}, 0\right), U\left(x_{1}, 0\right), \ldots, U\left(x_{N-1}, 0\right), U\left(x_{N}, 0\right)\right)^{T} .
\end{aligned}
$$

\section{Stability Analysis}

To investigate the stability analysis of the suggested algorithm, it is properly use Von-Neumann theory based on Fourier analysis. Supposing the quantity $U^{2}$ in the nonlinear term $U^{2} U_{x}$ is locally constant. Substituting the Fourier mode $\delta_{m}^{n}=\xi^{n} e^{i \sigma m h},(i=\sqrt{-1})$ into the form of (16) we attain,

$$
\begin{aligned}
& \xi^{n+1}\left(\eta_{1} e^{i(m-2) \theta}+\eta_{2} e^{i(m-1) \theta}+\eta_{3} e^{i m \theta}+\eta_{4} e^{i(m+1) \theta}+\eta_{5} e^{i(m+2) \theta}\right) \\
= & \xi^{n}\left(\eta_{5} e^{i(m-2) \theta}+\eta_{4} e^{i(m-1) \theta}+\eta_{3} e^{i m \theta}+\eta_{2} e^{i(m+1) \theta}+\eta_{1} e^{i(m+2) \theta}\right)
\end{aligned}
$$

where $\sigma$ is mode number, $h$ is the element size, $\theta=\sigma h$

$$
\begin{aligned}
& \eta_{1}=1-E Z_{m}-M, \quad \eta_{2}=26-10 E Z_{m}+2 M, \quad \eta_{3}=66, \\
& \eta_{4}=26+10 E Z_{m}-2 M, \quad \eta_{5}=1+E Z_{m}+M, \quad m=0,1, \ldots, N, \quad E=\frac{5}{2 h} \varepsilon \Delta t, M=\frac{30}{h^{3}} \mu \Delta t .
\end{aligned}
$$

If we simplify the Eq. (19),

$$
\xi=\frac{A+i B}{A-i B}
$$

is obtained where

$$
\begin{aligned}
& A=(52) \cos (\theta)+2 \cos (2 \theta)+66 \\
& B=\left(20 E Z_{m}-4 M\right) \sin (\theta)+\left(2 E Z_{m}+2 M\right) \sin (2 \theta) .
\end{aligned}
$$


According to the Fourier stability analysis, for the given scheme to be stable, the condition $|\xi|<1$ must be satisfied. Using a symbolic programming software or using simple calculations, since $a^{2}+b^{2}=a^{2}+(-b)^{2}$ it becomes evident that the modulus of $|\xi|$ is 1 . Therefore the linearized scheme is unconditionally stable.

\section{Numerical Results and Discussion}

In this part, to realise the correction of our algorithm, some numerical exercises have been calculated: the motion of single solitary wave whose analytical solution is known and extended the scheme to the study of two and three solitary waves, whose analytical solutions are unknown during the interaction. The initial boundary value problem (3) - (5) possesses following conservative quantities[29, 30];

$$
\begin{aligned}
& I_{1}=\int_{-\infty}^{\infty} U(x, t) d x, \\
& I_{2}=\int_{-\infty}^{\infty} U^{2}(x, t) d x, \\
& I_{3}=\int_{-\infty}^{\infty}\left[U^{4}(x, t)-\frac{6 \mu}{\varepsilon} U_{x}^{2}(x, t)\right] d x, \\
& I_{4}=\int_{-\infty}^{\infty}\left[U^{6}(x, t)-\frac{30 \mu}{\varepsilon} U^{2}(x, t) U_{x}^{2}(x, t)+\frac{18 \mu^{2}}{\varepsilon^{2}} U_{x x}^{2}(x, t)\right] d x .
\end{aligned}
$$

To calculate the difference between exact and numerical solutions at some specified times, $L_{2}$ and $L_{\infty}$ error norms

$$
L_{2}=\left\|U^{\text {exact }}-U_{N}\right\|_{2} \simeq \sqrt{h \sum_{J=0}^{N}\left|U_{j}^{\text {exact }}-\left(U_{N}\right)_{j}\right|^{2}}, L_{\infty}=\left\|U^{\text {exact }}-U_{N}\right\|_{\infty} \simeq \max _{j}\left|U_{j}^{\text {exact }}-\left(U_{N}\right)_{j}\right|
$$

have been used.

\subsection{The motion of single solitary wave}

For this problem, Eq.(3) is evaluated with the boundary conditions $U \rightarrow 0$ as $x \rightarrow \pm \infty$ and the initial condition

$$
U(x, 0)=A \sec h\left[k\left(x-x_{0}\right)\right]
$$

where $A=\sqrt{\frac{6 c}{\varepsilon}}, k=\sqrt{\frac{c}{\mu}}$ and $A$ is amplitude, $k$ is the width of the single solitary wave. The exact solution of the $\mathrm{MKdV}$ equation is given as

$$
U(x, t)=A \sec h\left[k\left(x-c t-x_{0}\right)\right]
$$

where $c$ and $x_{0}$ are arbitrary constants. For this problem, the exact values of the invariants can be given as [21]

$$
I_{1}=\pi \sqrt{\frac{6 \mu}{\varepsilon}}, I_{2}=\frac{12 \sqrt{\mu c}}{\varepsilon}, I_{3}=-\frac{64 c^{2}}{\varepsilon^{2}} \sqrt{\frac{\mu}{c}}, I_{4}=\frac{216 c^{2} \mu}{5 \varepsilon^{3}} \sqrt{\frac{c}{\mu}} .
$$

For this test problem, we have taken the parameters $\varepsilon=3, \mu=1, h=0.1, \Delta t=0.01, c=0.845$ and 0.3 through the interval $0 \leq x \leq 80$, so the solitary waves have amplitudes 1.3 and 0.7746 , respectively. The numerical algorithms are actuated to $t=1$ to get the error norms $L_{2}, L_{\infty}$ and conserved quantities $I_{1}, I_{2}, I_{3}$ and $I_{4}$. Invariants and error norms provided by the suggested method are given in Table (1) and Table (2). From these tables, it is obviously seen that the computed values of invariants are in good agreement with their analytical values. Solitary wave profiles are demonstrated at different time levels in Fig.(1) in which the soliton moves toward the right having nearly unchanged properties for example speed and amplitude.

\subsection{Interaction of two solitary waves}

As a second problem, we have studied the behavior of the interaction of two solitary waves having different amplitudes and travelling in the same direction. Initial condition of two well-seperated solitary waves of different amplitudes has the following form: 
Table 1. Invariants and error norms for single solitary wave with $\varepsilon=3, \mu=1, c=0.845, h=0.1$ and $\Delta t=0.01,0 \leq x \leq$ 80.

\begin{tabular}{ccccccc}
\hline$t$ & $I_{1}$ & $I_{2}$ & $I_{3}$ & $I_{4}$ & $L_{2}$ & $\mathrm{~L}_{\infty}$ \\
\hline 0.1 & 4.442863 & 3.676938 & 2.071325 & 1.050110 & $8.589 \mathrm{E}-02$ & $5.041 \mathrm{E}-02$ \\
0.2 & 4.442864 & 3.676939 & 2.071323 & 1.050109 & $1.716 \mathrm{E}-01$ & $1.006 \mathrm{E}-01$ \\
0.3 & 4.442865 & 3.676939 & 2.071323 & 1.050108 & $2.569 \mathrm{E}-01$ & $1.506 \mathrm{E}-01$ \\
0.4 & 4.442866 & 3.676938 & 2.071322 & 1.050107 & $3.418 \mathrm{E}-01$ & $2.001 \mathrm{E}-01$ \\
0.5 & 4.442863 & 3.676937 & 2.071322 & 1.050107 & $4.259 \mathrm{E}-01$ & $2.490 \mathrm{E}-01$ \\
0.6 & 4.442861 & 3.676937 & 2.071319 & 1.050105 & $5.091 \mathrm{E}-01$ & $2.971 \mathrm{E}-01$ \\
0.7 & 4.442861 & 3.676935 & 2.071318 & 1.050104 & $5.913 \mathrm{E}-01$ & $3.446 \mathrm{E}-01$ \\
0.8 & 4.442862 & 3.676934 & 2.071316 & 1.050102 & $6.724 \mathrm{E}-01$ & $3.907 \mathrm{E}-01$ \\
0.9 & 4.442863 & 3.676932 & 2.071314 & 1.050101 & $7.521 \mathrm{E}-01$ & $4.363 \mathrm{E}-01$ \\
1.0 & 4.442863 & 3.676933 & 2.071312 & 1.050100 & $8.304 \mathrm{E}-01$ & $4.805 \mathrm{E}-01$ \\
\hline
\end{tabular}

Table 2. Invariants and error norms for single solitary wave with $\varepsilon=3, \mu=1, c=0.3, h=0.1$ and $\Delta t=0.01,0 \leq x \leq 80$.

\begin{tabular}{ccccccc}
\hline$t$ & $I_{1}$ & $I_{2}$ & $I_{3}$ & $I_{4}$ & $L_{2}$ & $\mathrm{~L}_{\infty}$ \\
\hline 0.1 & 4.442792 & 2.190881 & 0.4381725 & 0.078951 & $1.403 \mathrm{E}-02$ & $6.361 \mathrm{E}-03$ \\
0.2 & 4.442779 & 2.190881 & 0.4381727 & 0.078970 & $2.807 \mathrm{E}-02$ & $1.272 \mathrm{E}-02$ \\
0.3 & 4.442776 & 2.190881 & 0.4381729 & 0.078994 & $4.210 \mathrm{E}-02$ & $1.907 \mathrm{E}-02$ \\
0.4 & 4.442777 & 2.190880 & 0.4381731 & 0.079025 & $5.613 \mathrm{E}-02$ & $2.543 \mathrm{E}-02$ \\
0.5 & 4.442771 & 2.190882 & 0.4381731 & 0.079066 & $7.906 \mathrm{E}-02$ & $7.016 \mathrm{E}-02$ \\
0.6 & 4.442772 & 2.190881 & 0.4381735 & 0.079120 & $8.418 \mathrm{E}-02$ & $3.814 \mathrm{E}-02$ \\
0.7 & 4.442766 & 2.190881 & 0.4381731 & 0.079190 & $9.819 \mathrm{E}-02$ & $4.448 \mathrm{E}-02$ \\
0.8 & 4.442769 & 2.190880 & 0.4381730 & 0.079281 & $1.121 \mathrm{E}-01$ & $5.082 \mathrm{E}-02$ \\
0.9 & 4.442766 & 2.190881 & 0.4381731 & 0.079399 & $1.261 \mathrm{E}-01$ & $5.714 \mathrm{E}-02$ \\
1.0 & 4.442765 & 2.190882 & 0.4381731 & 0.079551 & $1.401 \mathrm{E}-01$ & $6.345 \mathrm{E}-02$ \\
\hline
\end{tabular}

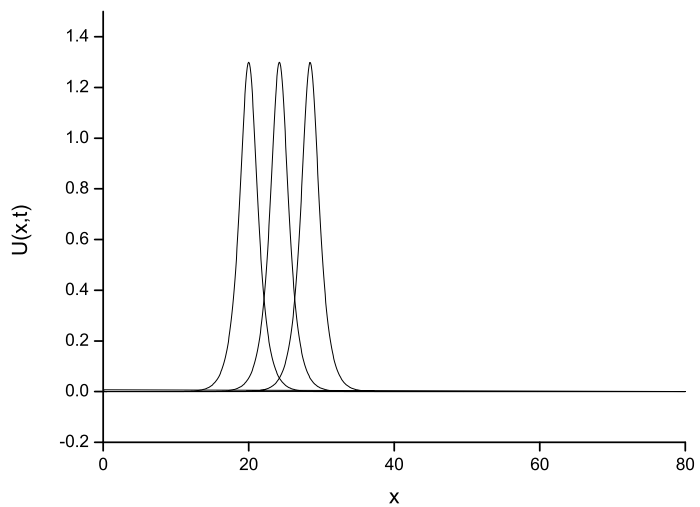

Figure 1. Single solitary wave with $\varepsilon=3, \mu=1, c=0.845, h=0.1, \Delta t=0.01$ and $0 \leq x \leq 80$ at $t=0,5$ and 10 .

$$
U(x, 0)=\sum_{j=1}^{2} A_{j} \sec h\left[c_{j}\left(x-x_{j}\right)\right]
$$

$(j=1,2) c_{j}$ and $x_{j}$ are arbitrary constants. We have chosen the parameters $\varepsilon=3, \mu=1, h=0.1, \Delta t=0.01$, $c_{1}=2, c_{2}=1, x_{1}=15$ and $x_{2}=25$ over the interval $0 \leq x \leq 80$. The run of the algorithm is carried up to time 
$t=5$ to obtain the values of the invariants. The obtained results are tabulated in Table (3). Table (3) shows that invariants are nearly constant as the time progresses. The calculated quantities $I_{1}, I_{2}, I_{3}$ and $I_{4}$ change by less than $2.16 \times 10^{-3}, 6.1 \times 10^{-3}, 4.95 \times 10^{-2}$ and $2.49 \times 10^{-2}$, respectively. Therefore, we can say our method is marginally conservative. The interaction of two solitary wave is depicted at different time levels in Figure (2).

Table 3. Invariants for the interaction of two solitary waves with $\varepsilon=3, \mu=1, h=0.1, \Delta t=0.01, c_{1}=2, c_{2}=1, x_{1}=15$ and $x_{2}=25,0 \leq x \leq 80$.

\begin{tabular}{ccccc}
\hline$t$ & $I_{1}$ & $I_{2}$ & $I_{3}$ & $I_{4}$ \\
\hline 0 & 8.885730 & 9.659342 & 10.219180 & 10.668990 \\
1 & 8.885097 & 9.657387 & 10.211370 & 10.653420 \\
2 & 8.884507 & 9.655479 & 10.203760 & 10.638260 \\
3 & 8.883841 & 9.653700 & 10.196620 & 10.624040 \\
4 & 8.883119 & 9.652200 & 10.190490 & 10.611900 \\
5 & 8.882933 & 9.651279 & 10.186450 & 10.603890 \\
\hline
\end{tabular}
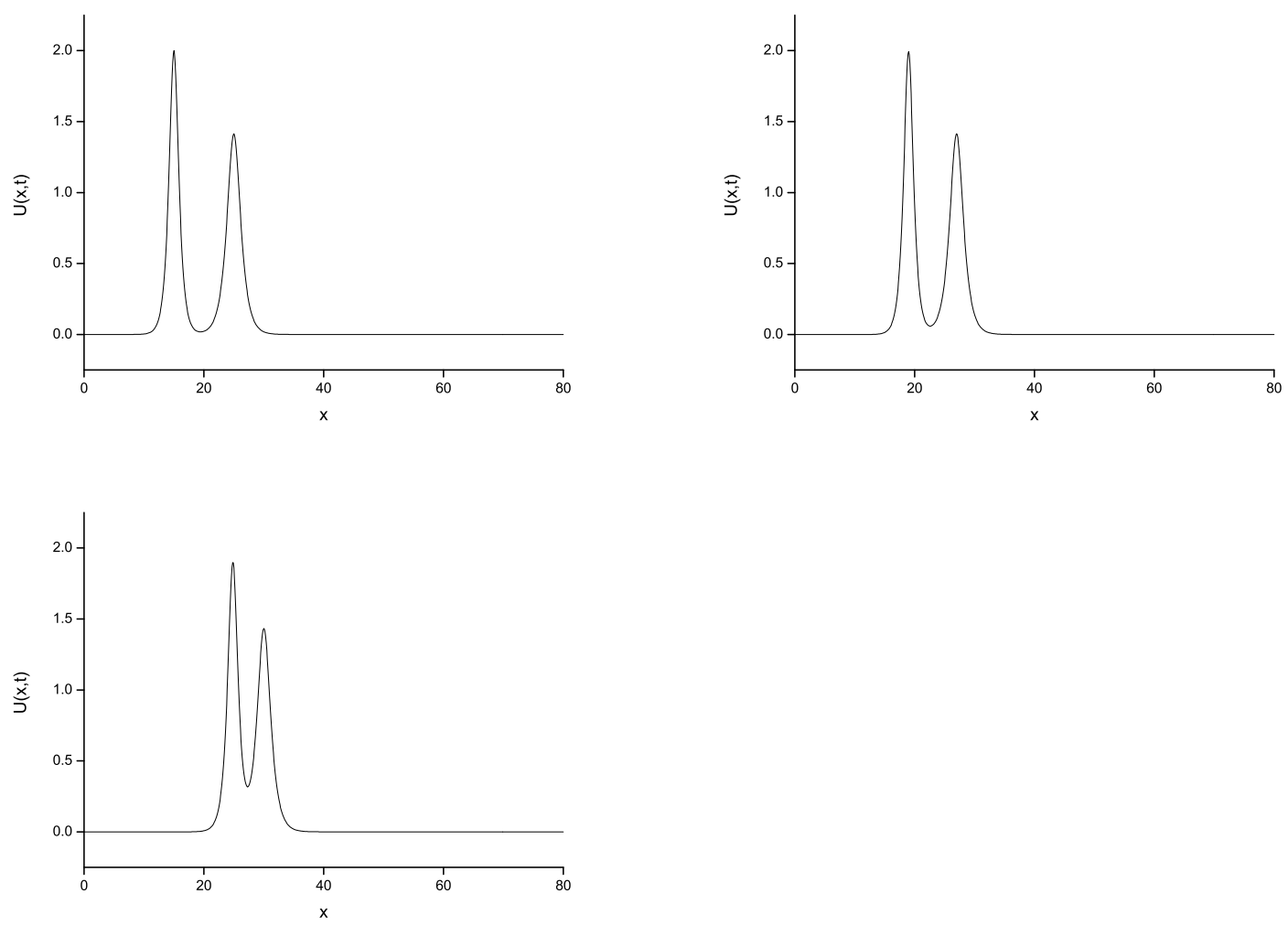

Figure 2. Interaction of two solitary waves with $\varepsilon=3, \mu=1, h=0.1, \Delta t=0.01, c_{1}=2, c_{2}=1, x_{1}=15, x_{2}=25$ and $0 \leq x \leq 80$ at $t=0,2$ and 5 .

\subsection{Interaction of three solitary waves}

As a last problem, we have considered the behavior of the interaction of three solitary waves having different amplitudes and traveling in the same direction. We examined the interaction of three solitary waves by using the initial condition 


$$
U(x, 0)=\sum_{j=1}^{3} A_{j} \sec h\left[c_{j}\left(x-x_{j}\right)\right]
$$

together with boundary conditions $U \rightarrow 0$ as $x \rightarrow \pm \infty$. We have conceived the problem with parameters $\varepsilon=3$, $\mu=1, h=0.1, \Delta t=0.01, c_{1}=2, c_{2}=1, c_{3}=0.5, x_{1}=15, x_{2}=25$ and $x_{3}=35$ over the interval $0 \leq x \leq 80$. The experiment is run from $t=0$ to $t=5$ and values of the quantities are listed in Table (4). Table (4) indicates that invariants are nearly constant as the time increases. The behavior of the interaction of three solitary waves denotes at different times in Figure (3).

Table 4. Invariants for the interaction of three solitary waves with $\varepsilon=3, \mu=1, h=0.1, \Delta t=0.01, c_{1}=2, c_{2}=1, c_{3}=0.5$, $x_{1}=15, x_{2}=25$ and $x_{3}=35,0 \leq x \leq 80$.

\begin{tabular}{ccccc}
\hline$t$ & $I_{1}$ & $I_{2}$ & $I_{3}$ & $I_{4}$ \\
\hline 0 & 13.328670 & 12.519940 & 11.228390 & 11.019630 \\
1 & 13.328050 & 12.518010 & 11.220640 & 11.004170 \\
2 & 13.327570 & 12.516120 & 11.213060 & 10.989010 \\
3 & 13.326950 & 12.514400 & 11.206050 & 10.975050 \\
4 & 13.325990 & 12.513020 & 11.200220 & 10.963460 \\
5 & 13.326010 & 12.512290 & 11.196690 & 10.956380 \\
\hline
\end{tabular}
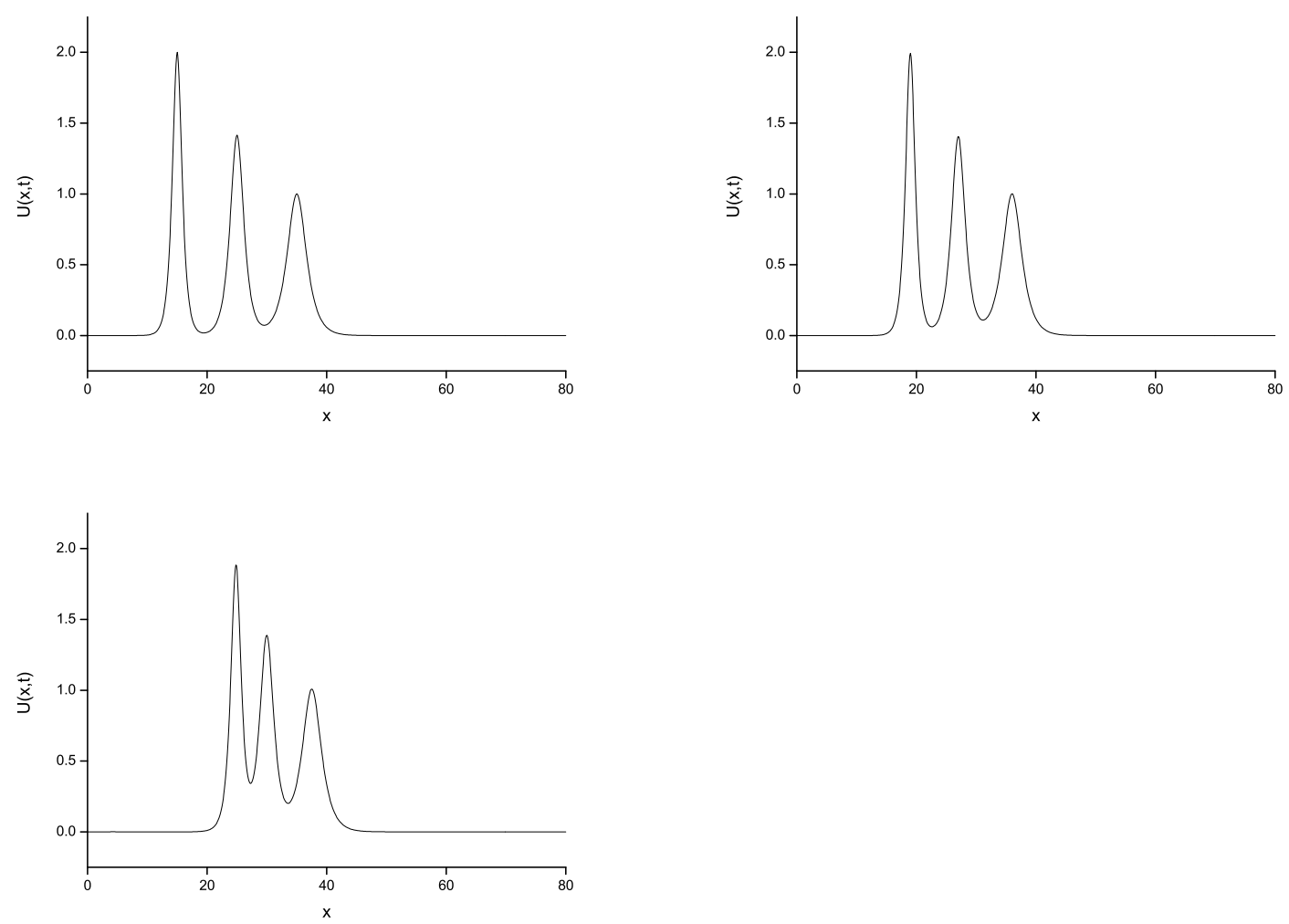

Figure 3. Interaction of three solitary waves with $\varepsilon=3, \mu=1, h=0.1, \Delta t=0.01, c_{1}=2, c_{2}=1, c_{3}=0.5, x_{1}=15$, $x_{2}=25, x_{3}=35$ and $0 \leq x \leq 80$ at $t=0,2$ and 5 . 


\section{Conclusion}

In this article, we have applied a quartic B-spline subdomain finite element method to the MKdV equation. Three different test problems have been solved. To demonstrate the efficiency of numerical scheme, the error norms $L_{2}$, $L_{\infty}$ and conserved quantities $I_{1}, I_{2}, I_{3}$ and $I_{4}$ have been calculated for the test problems. According to the tables in the paper, one can has easily seen that our error norms are enough small and the obtained invariants are acceptable in good agreement with their exact values. Our numerical scheme is also unconditionally stable. So, we can say our numerical algorithm is a reliable method for getting the numerical solutions of the physically important non-linear partial differential equations.

\section{REFERENCES}

1. D. J. Korteweg, and G. de Vries, On the change of form of long waves advancing in a rectangular canal, and a new type of long stationary wave, Philosophical Magazine, vol. 39, pp. 422-443, 1895.

2. N. J. Zabusky, A synergetic approach to problem of nonlinear dispersive wave propagation and interaction, in: W. Ames (Ed.), Proc. Symp. Nonlinear Partial Diff. Equations, Academic Press, pp. 223-258, 1967.

3. B. Fornberg, and G.B. Whitham, A numerical and theoretical study of certain nonlinear wavephenomena, Philos. Trans. Roy. Soc. vol. 289, pp. 373-404, 1978.

4. N. J. Zabusky, and M. D. Kruskal, Interaction of solitons in a collisionless plasma and the recurrence of initial states, Phys. Rev. Lett., vol. 5, no. 6, pp. 240-243, 1965.

5. C. S Gardner, J. M. Green, M. D. Kruskal, and R. M. Miura, Method for solving Korteweg- de Vries equation, Phys. Rev., vol. 19, pp. 1095-1100, 1967.

6. K. Goda, On instability of some finite difference schemes for Korteweg-de Vries equation, J.Phys. Soc. Japan, vol. 39, pp. 229-236, 1975.

7. A. C. Vliengenthart, On finite difference methods for the Korteweg-de Vries equation, J. Eng. Math., vol. 5, pp. 137-155, 1971.

8. A. A. Soliman, Collocation solution of the Korteweg-De Vries equation using septic splines, Int. J. Comput. Math. vol. 81, pp. 325-331, 2004

9. D. Irk, İ. Dağ, and B. Saka, A small time solutions for the Korteweg-de Vries equation using spline approximation, Appl. Math. Comput., vol. 173 , no. 2, pp. 834-846, 2006.

10. A. Canıvar, M. Sarı and I. Dağ,, A Taylor-Galerkin finite element method for the KdV equation using cubic B-splines, Physica B, vol. 405, pp. 3376-3383, 2010.

11. A. Korkmaz, Numerical algorithms for solutions of Korteweg-de Vries Equation, Numerical Methods for Partial Differential Equations, vol. 26, no. 6, pp. 1504-1521, 2010.

12. Ö. Ersoy, and I. Da , Cubic B-Spline Algorithm for Korteweg-de Vries Equation, Advances in Numerical Analysis, vol. 2015, pp. $1-8,2015$

13. E. N. Aksan, and A. Ozdes, Numerical solution of Korteweg-de Vries equation by Galerkin B-spline finite element method, Applied Mathematics and Computation, vol. 175, pp. 1256-1265, 2006.

14. D. Irk, Quintic B-spline Galerkin method for the KdV equation, Anadolu University Journal of Science and Technology BTheoritical Sciences, vol. 5, no. 2, pp. 111-119, 2017.

15. B. Saka, Cosine expansion-based differential quadrature method for numerical solution of the KdV equation, Chaos Soliton Fract. vol. 40, pp. 2181-2190, 2009.

16. S. B.G. Karakoc, and H. Zeybek, A cubic B-spline Galerkin approach for the numerical simulation of the GEW equation, Stat., Optim. Inf. Comput., Vol. 4, 2016, pp 30-41.

17. B.G. Karakoc, H. Zeybek, and T. Ak, Numerical solutions of the Kawahara equation by the septic B-spline collocation method Stat., Optim. Inf. Comput., Vol. 2, 2014, pp 211-221.

18. J. M. Heris, and I. Zamanpour, Exact travelling wave solutions of the symmetric regularized long wave (SRLW) using analytical methods, Stat., Optim. Inf. Comput., Vol. 2, March 2014, pp 47-55.

19. S. Kutluay, A. R. Bahadır, and A. Ozdes, A small time solutions for the Korteweg-de Vries equation, Appl. Math. Comput. vol. 107, pp. 203-210, 2000

20. D. Kaya, An application for the higher order modified KdV equation by decomposition method, Commun. in Nonlinear Science and Num. Simul., vol. 10, pp. 693-702, 2005.

21. A. Biswas, and K. R .Raslan, Numerical simulation of the modified Korteweg-de Vries Equation, Physics of Wave Phenomena, vol 19, no. 2, pp. 142-147, 2011.

22. K. R. Raslan, and H. A. Baghdady, A finite difference scheme for the modified Korteweg-de Vries equation, General Mathematics Notes, vol. 27, no. 1, pp. 101-113, 2015

23. K. R. Raslan, and H. A. Baghdady, New algorithm for solving the modified Korteweg-de Vries (mKdV) equation, International Journal of Research and Reviews in Applied Sciences, vol. 18, no. 1, pp. 59-64, 2014.

24. A. M. Wazwaz, A variety of (3+1)-dimensional $m K d V$ equations derived by using the $m K d V$ recursion operator, Computers and Fluids, vol. 93, no. 10, pp. 41-45, 2014.

25. A. M. Wazwaz, New (3+1)-dimensional nonlinear evolution equations with mKdV equation constituting its main part: multiple soliton solutions, Chaos, Solitons and Fractals, vol. 76, pp. 93-97, 2015. 
26. T. Ak, S. B. G. Karakoc, and A. Biswas, A New Approach for Numerical Solution of Modified Korteweg-de Vries Equation, Iran J. Sci. Technol. Trans. Sci., vol. 41, pp. 1109-1121, 2017.

27. T. Ak, S. B. G. Karakoc, and A. Biswas, Application of Petrov-Galerkin nite element method to shallow water waves model: Modified Korteweg-de Vries equation, Scientia Iranica B., vol. 24, no. 3, pp. 1148-1159, 2017.

28. P. M. Prenter, Splines and Variational Methods, John Wiley \& Sons, New York, NY.USA, 1975.

29. R. M. Miura, C. S. Gardner, and M. D. Kruskal, Korteweg-de Vries equation and generalizations. II. Existence of conservation laws and constants of motion, J. Math. Phys., vol. 9, no. 8,1204, 1968.

30. R. M. Miura, The Korteweg-de Vries equation: a survey of results, SIAM Rev., vol.18, no.3, pp. 412-459, 1976. 